\title{
Approximating the 2-Machine Flow Shop Problem with Exact Delays Taking Two Values
}

\author{
Alexander Ageev
}

\begin{abstract}
In the 2-Machine Flow Shop problem with exact delays the operations of each job are separated by a given time lag (delay). Leung et al. (2007) established that the problem is strongly NP-hard when the delays may have at most two different values. We present further results for this case: we prove that the existence of $(1.25-\varepsilon)$-approximation implies $\mathrm{P}=\mathrm{NP}$ and develop a 2 -approximation algorithm.
\end{abstract}

\section{Introduction}

An instance of the 2-Machine Flow Shop problem with exact delays consists of $n$ triples $\left(a_{j}, l_{j}, b_{j}\right)$ of nonnegative integers where $j$ is a job in the set of jobs $J=\{1, \ldots, n\}$. Each job $j$ must be processed first on machine 1 and then on machine $2, a_{j}$ and $b_{j}$ are the lengths of operations on machines 1 and 2 , respectively. The operation of job $j$ on machine 2 must start exactly $l_{j}$ time units after the operation on machine 1 has been completed. The goal is to minimize makespan. In the standard three-field notation scheme the problem is written as $F 2 \mid$ exact $l_{j} \mid C_{\text {max }}$.

One of evident applications of scheduling problems with exact delays is chemistry manufacturing where there often may be an exact technological delay between the completion time of some operation and the initial time of the next operation. The problems with exact delays also arise in command-and-control applications in which a centralized commander distributes a set of orders (associated with the first operations) and must wait to receive responses (corresponding to the second operations) that do not conflict with any other (for more extensive discussion on the subject, see [5, 9]). Condotta [4] describes an application related to booking appointments of chemotherapy treatments [4]. 
The approximability of $F 2 \mid$ exact $l_{j} \mid C_{\max }$ was studied by Ageev and Kononov in [2]. They proved that the existence of $(1.5-\varepsilon)$-approximation algorithm implies $\mathrm{P}=\mathrm{NP}$ and constructed a 3 -approximation algorithm. They also give a 2-approximation algorithm for the cases when $a_{j} \leq b_{j}$ and $a_{j} \geq b_{j}$, $j \in J$. These algorithms were independently developed by Leung et al. in [8]. The case of unit processing times $\left(a_{j}=b_{j}=1\right.$ for all $\left.j \in J\right)$ was shown to be strongly NP-hard by $\mathrm{Yu}$ [10, 11]. Ageev and Baburin [1] gave a 1.5-approximation algorithm for solving this case.

In this paper we consider the case when $l_{j} \in\left\{L_{1}, L_{2}\right\}$ for all $j \in\{1, \ldots, n\}$. In the three-field notation scheme this case can be written as $F 2 \mid$ exact $l_{j} \in$ $\left\{L_{1}, L_{2}\right\} \mid C_{\max }$. The problem was shown to be strongly NP-hard by Leung et al. [8].

Our results are the following: we prove that the existence of $(1.25-\varepsilon)$ approximation for $F 2 \mid$ exact $l_{j} \in\{0, L\} \mid C_{\max }$ implies $\mathrm{P}=\mathrm{NP}$ and present a 2-approximation algorithm for $F 2 \mid$ exact $l_{j} \in\left\{L_{1}, L_{2}\right\} \mid C_{\max }$.

Throughout the paper we use the standard notation: $C_{\max }(\sigma)$ stands for the length of a schedule $\sigma$ (makespan); $C_{\max }^{*}$ means the length of a shortest schedule.

We assume that no job has a missing operation in the sense that a zero processing time implies that the job has to visit the machine for an infinitesimal amount of time $\delta>0$.

\section{Inapproximability lower bound for $F 2 \mid$ exact $l_{j} \in\{0, L\} \mid C_{\max }$}

In this section we establish the inapproximability lower bound for the case $F 2 \mid$ exact $l_{j} \in\{0, L\} \mid C_{\max }$, i.e., when $L_{1}=0, L_{2}=L$. To this end consider the following reduction from PARTITION problem.

\section{PARTITION}

Instance: Nonnegative integers $w_{1}, \ldots, w_{m}$ such that $\sum_{k=1}^{m} w_{k}=2 S$.

Question: Does there exist a subset $X \subseteq\{1, \ldots, m\}$ such that $\sum_{k \in X} w_{k}$ $=S ?$

Consider an instance $\mathcal{I}$ of PARTITION and construct an instance $\mathcal{I}^{\prime}$ of $F 2 \mid$ exact $l_{j} \in\{0, L\} \mid C_{\max }$. 
Let $R>5 S$. Set $J=\{1, \ldots, m+6\}$ and

$$
\begin{aligned}
a_{k} & =b_{k}=w_{k}, l_{k}=2 R \text { for } \quad k=1, \ldots m, \\
a_{m+1} & =b_{m+1}=R, l_{m+1}=0, \\
a_{m+2} & =b_{m+2}=R, l_{m+2}=2 R, \\
a_{m+3} & =0, b_{m+3}=R-S, l_{m+3}=0, \\
a_{m+4} & =R-S, b_{m+4}=0, l_{m+4}=0, \\
a_{m+5} & =0, b_{m+5}=R, l_{m+5}=0, \\
a_{m+6} & =R, b_{m+6}=0, l_{m+6}=0 .
\end{aligned}
$$

We will refer to the jobs in $\{1, \ldots, m\}$ as small and to the remaining six jobs as big.

\section{Lemma 1.}

(i) If $\sum_{k \in X} w_{k}=S$ for some subset $X \subseteq\{1, \ldots m\}$, then there exists a feasible schedule $\sigma$ such that $C_{\max }(\sigma) \leq 4 R+4 S$.

(ii) If there exists a feasible schedule $\sigma$ such that $C_{\max }(\sigma) \leq 4 R+4 S$, then $\sum_{k \in X} w_{k}=S$ for some set $X \subseteq\{1, \ldots m\}$.

(iii) If $C_{\max }(\sigma)>4 R+4 S$ for any feasible schedule $\sigma$, then $C_{\max }^{*} \geq 5 R-S$.

Proof. (i) Let $X \subseteq\{1, \ldots, m\}$ such that $\sum_{k \in X} w_{k}=S$. Then $\sum_{k \in Y} w_{k}=S$ where $Y=\{1, \ldots, m\} \backslash X$.

First of all point out that the whole construction presenting a feasible schedule can be moved along the time line in both directions. So the length of the schedule is the length of the time interval between the starting time of the first operation (which is not necessarily equal to zero) and the completion time of the last one. To construct the required schedule arrange the big jobs

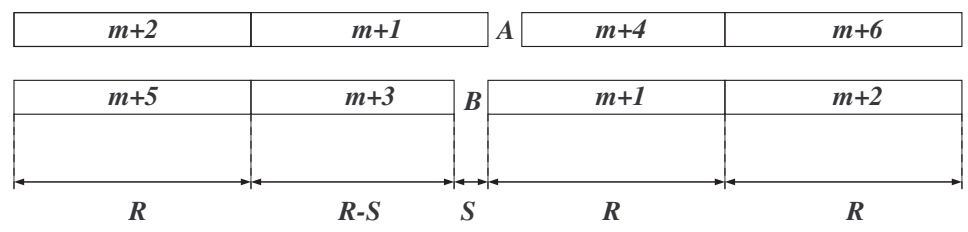

Figure 1: Scheduling the big jobs.

in the order shown in Fig. 1. This construction has two idle intervals: $A$ on machine 1 and $B$ on machine 2. The interval $A$ is between the end of the 
first operation of job $m+1$ and the beginning of the first operation of job $m+4$. The interval $B$ is between the end of the second operation of job $m+3$ and the beginning of the second operation of job $m+1$. Both intervals have length $S$.

For scheduling the small jobs we use the following rule. Schedule the small jobs in $X$ in such a way that their first operations are executed within the time interval $A$ in non-decreasing order of the lengths. Correspondingly, w.l.o.g. we may assume that $X=\{1,2, \ldots, q\}$ and $w_{1} \leq w_{2} \leq \ldots \leq w_{q}$.

Denote by $A^{\prime}$ the time interval between the end of the second operation of job $m+2$ and the end of the last operation of job $q$. It is easy to understand (see Fig. 2) that all the second operations of jobs $\{1, \ldots, q\}$ fall within $A^{\prime}$ and the length of $A^{\prime}$ is equal to

$$
\sum_{i=1}^{q} w_{i}+w_{1}+\left(w_{2}-w_{1}\right)+\left(w_{3}-w_{2}\right)+\ldots+\left(w_{q}-w_{q-1}\right)=\sum_{i=1}^{q} w_{i}+w_{q},
$$

which does not exceed $2 S$. Now we observe that the construction is sym-

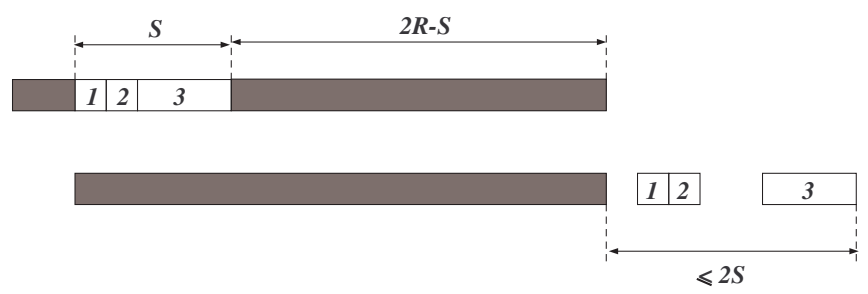

Figure 2: Scheduling the small jobs in $X$.

metric and schedule the jobs in $Y$ quite similarly. More precisely, the second operations of jobs in $Y$ are executed without interruption within the time interval $B$ in non-increasing order of their lengths. Then the first operations of these jobs fall within a time interval of length at most $2 S$.

Finally, we arrive at the schedule shown in Fig. 3. From the above argument its length does not exceed $4 R+4 S$, as required.

(ii) Let $\sigma$ be a feasible schedule with $C_{\max }(\sigma) \leq 4 R+4 S$. Observe first that in any schedule of length at most $4 R+4 S$ both operations of job $m+1$ are executed exactly within the lag time interval of job $m+2$, since otherwise $C_{\max }(\sigma) \geq 5 R$. So for these jobs we have the initial construction shown in Fig. 4 . Denote by $t_{0}, t_{1}, t_{2}, t_{3}, t_{4}$ the junction times of the operations of these jobs (see Fig. 4).

Observe that the schedule $\sigma$ has the following property (Q): for any small job either it completes at time not earlier than $t_{1}$, or starts at time not later 


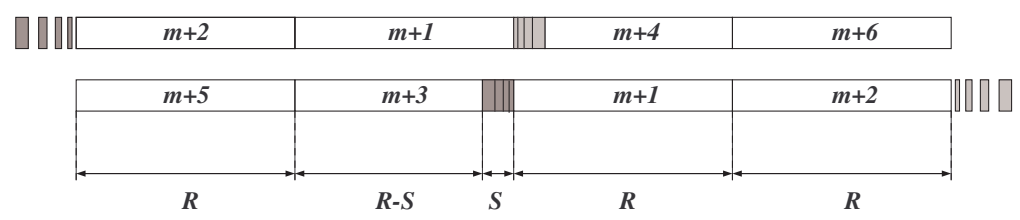

Figure 3: The jobs in $\{1, \ldots, m\}$ are executed within the shaded intervals.

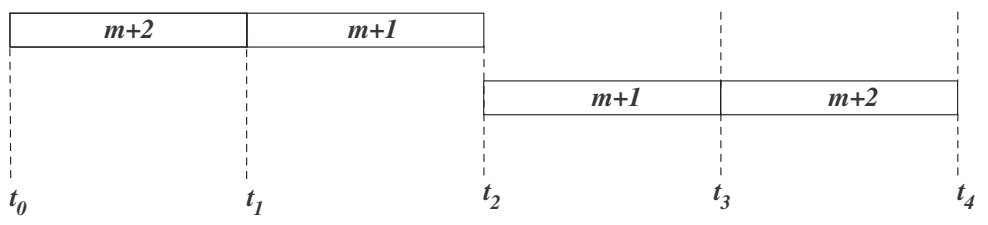

Figure 4: The initial construction.

than $t_{3}$. It follows from the fact that otherwise the length of $\sigma$ is at least $5 R$. Let $X$ be the subset of small jobs that complete at time not earlier than $t_{1}$. Then $Y=\{1, \ldots, m\} \backslash X$ is the subset of small jobs that start at time not later than $t_{3}$. For definiteness assume that $X \neq \emptyset$. This immediately implies that job $m+5$ starts at time not later than $t_{0}$. Then job $m+3$ starts exactly at time $t_{1}$, since otherwise the length of $\sigma$ is at least $5 R-S$, which is greater than $4 R+4 S$ due to the choice of $R$ and $S$. Thus the second operations of the jobs in $X$ are executed within the interval $\left[t_{2}-S, S\right]$. It follows that $Y \neq \emptyset$. Moreover, a similar argument shows that the first operations of the jobs in $Y$ are executed within the interval $\left[t_{2}, t_{2}+S\right]$. Thus we have $\sum_{j \in X} w_{j} \leq S$ and $\sum_{j \in Y} w_{j} \leq S$, which implies $\sum_{j \in X} w_{j}=\sum_{j \in Y} w_{j}=S$, as required.

(iii) Let $\sigma$ be a feasible schedule. By the assumption of (iii) $C_{\max }(\sigma)>$ $4 R+4 S$. We may assume that $\sigma$ contains the initial construction and satisfies property (Q) (see (ii)), since otherwise we are done. Let $X$ and $Y$ be defined as in (ii). From (i) it follows that $\sum_{j \in X} w_{j} \neq \sum_{j \in Y} w_{j}$. W.l.o.g. we may assume that $\sum_{j \in X} w_{j}>\sum_{j \in Y} w_{j}$, i.e., $\sum_{j \in X} w_{j}>S$. Then by property (Q) neither job $m+3$ nor job $m+5$ starts at time $t_{1}$. It follows that at least one of these jobs is executed outside the interval $\left[t_{0}, t_{4}\right]$, which implies $C_{\max }(\sigma) \geq 5 R-S$ (one of the possible configurations is shown in Fig. 5).

Set $R=k S$. Then $5 R-S=S(5 k-1)$. On the other hand, $4 R+4 S=$ 


\begin{tabular}{|c|c|c|c|c|}
\hline & $m+2$ & $m+1$ & & \\
\hline$m+3$ & $m+5$ & & $m+1$ & $m+2$ \\
\hline
\end{tabular}

Figure 5: One of the possible configurations for (iii).

$4 k S+4 S=S(4 k+4)$. The fraction

$$
\frac{5 k-1}{4 k+4}
$$

tends to 1.25 as $k$ tends to infinity. Thus Lemma 1 implies

Theorem 1. If the problem $F 2 \mid$ exact $l_{j} \in\{0, L\} \mid C_{\max }$ admits $a(1.25-\varepsilon)$ approximation algorithm, then $P=N P$.

\section{A 2-approximation algorithm for $F 2 \mid$ exact $l_{j} \in\left\{L_{1}, L_{2}\right\} \mid C_{\max }$}

In this section we present a simple 2-approximation algorithm for solving $F 2 \mid$ exact $l_{j} \in\left\{L_{1}, L_{2}\right\} \mid C_{\max }$.

We show first that the case when the delays are the same for all jobs $\left(L_{1}=L_{2}=L\right)$ is polynomially solvable. Note that any feasible schedule $\sigma$ of an instance of $F 2 \mid$ exact $l_{j}=L \mid C_{\max }$ can be associated with a feasible schedule $\sigma^{\prime}$ of the corresponding instance of $F 2 \mid$ exact $l_{j}=0 \mid C_{\max }$ and their lengths satisfy $C_{\max }(\sigma)=C_{\max }\left(\sigma^{\prime}\right)+L$. More precisely, shifting the second operations of all jobs to the left by distance $L$ gives a feasible schedule to the problem with zero delays, and vise versa (see Fig. 6). The problem $F 2 \mid$ exact $l_{j}=0 \mid C_{\max }$ (all delays are equal to 0 ) is nothing but the 2machine no-wait Flow Shop problem. The latter problem is known to be solvable in $O(n \log n)$ time [6, 7, 3]. Therefore the problem $F 2 \mid$ exact $l_{j}=$ $L \mid C_{\max }$ is solvable in $O(n \log n)$ time for all $L \geq 0$.

Let $\mathcal{I}_{1}, \mathcal{I}_{2}$ be instances of $F 2 \mid$ exact $l_{j} \mid C_{\text {max }}$ with disjoint sets of jobs $J_{1}$ and $J_{2}$. Let $\sigma_{k}, k=1,2$, be feasible schedules of $\mathcal{I}_{k}$, respectively. Consider an instance $\mathcal{I}$ of $F 2 \mid$ exact $l_{j} \mid C_{\max }$ formed by the union of $J_{1}$ and $J_{2}$. Denote by $\sigma_{1} \oplus \sigma_{2}$ the schedule of $\mathcal{I}$ obtained from $\sigma_{1}$ and $\sigma_{2}$ by concatenation of schedules $\sigma_{1}$ and $\sigma_{2}$. More precisely, the schedule $\sigma_{1} \oplus \sigma_{2}$ first executes the jobs in $J_{1}$ according to the schedule $\sigma_{1}$ and then as earlier as possible starts 
(a)

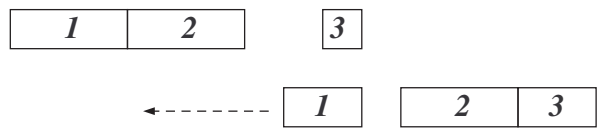

(b)

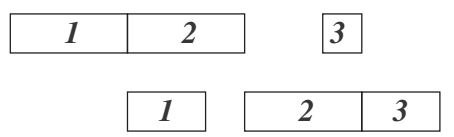

Figure 6: (a) A schedule with the same delay $L$ for all jobs; (b) the corresponding schedule with delay 0 for all jobs.

executing the jobs in $J_{2}$ according to the schedule $\sigma_{2}$. An example with $J_{1}=\{(3,2,3),(2,2,4)\}$ and $J_{2}=\{(4,0,2),(5,0,1)\}$ is depicted in Fig. 7.

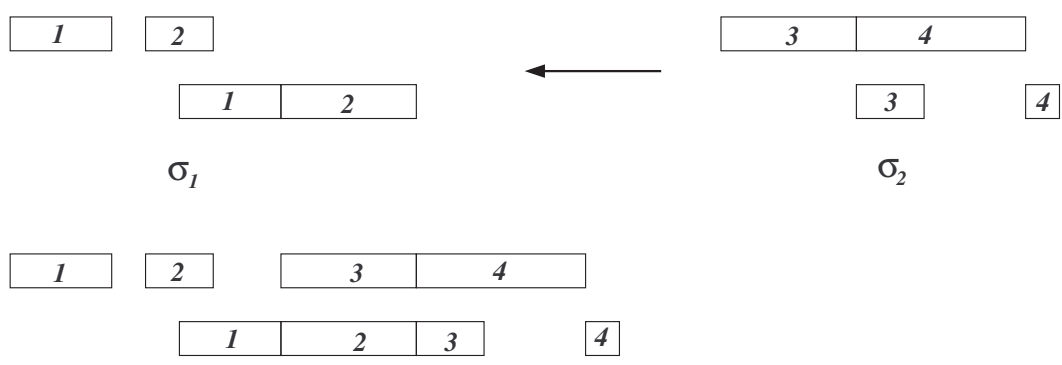

Figure 7: The schedule shown below is the concatenation of $\sigma_{1}$ and $\sigma_{2}$.

We now give a description of an approximation algorithm for the problem $F 2 \mid$ exact $l_{j} \in\left\{L_{1}, L_{2}\right\} \mid C_{\max }$.

\section{Algorithm Concatenation}

Input: An instance $\left\{\left(a_{j}, l_{j}, b_{j}\right): j \in J\right\}, l_{j} \in\left\{L_{1}, L_{2}\right\}$.

Output: A feasible schedule $\sigma$.

1. Set $J_{k}=\left\{j \in J: l_{j}=L_{k}, k=1,2\right\}$. For $k=1,2$ form the instances $\mathcal{I}_{k}=\left\{\left(a_{j}, L_{k}, b_{j}\right): j \in J_{k}\right\}$ of $F 2 \mid$ exact $l_{j}=L \mid C_{\max }$.

2. Solve the instances $\mathcal{I}_{k}, k=1,2$. Let $\sigma_{k}, k=1,2$, be optimal schedules of $\mathcal{I}_{k}$, respectively.

3. Set $\sigma=\sigma_{1} \oplus \sigma_{2}$.

As mentioned above the time complexity of Step 2 is $O(n \log n)$. So the overall running time of Algorithm CONCATENATION is $O(n \log n)$.

The approximation bound is derived from the following easy lemmata. 
Lemma 2. Let $C_{\max }^{*}$ be the length of an optimal schedule to the instance $\left\{\left(a_{j}, l_{j}, b_{j}\right): j \in J\right\}, l_{j} \in\left\{L_{1}, L_{2}\right\}$. Then $C_{\max }\left(\sigma_{k}\right) \leq C_{\max }^{*}$ for $k=1,2$.

Proof. Evident.

Lemma 3. $C_{\max }(\sigma) \leq C_{\max }\left(\sigma_{1}\right)+C_{\max }\left(\sigma_{2}\right)$.

Proof. Follows from the definition of operation $\oplus$.

From Lemmata 2 and 3 we have

$$
C_{\max }(\sigma) \leq 2 C_{\max }^{*}
$$

Thus we arrive at the following

Theorem 2. Algorithm CONCATENATION runs in time $O(n \log n)$ and finds a feasible schedule of $F 2 \mid$ exact $l_{j} \in\left\{L_{1}, L_{2}\right\} \mid C_{\max }$ whose length is within a factor of 2 of the optimum.

\section{Tightness.}

For $a>\delta>0$, consider the instance $\left\{\left(a_{j}, l_{j}, b_{j}\right): j \in J\right\}, l_{j} \in\left\{L_{1}, L_{2}\right\}$, consisting of $k$ jobs $(a, 0, a)$ and a single job $(\delta,(k+1) a, \delta)$. It is clear that

$$
C_{\max }^{*}=(k+1) a+2 \delta
$$

(see Fig. 8 for the optimal schedule). Let $\sigma_{\text {conc }}$ denote the schedule returned by Algorithm Concatenation. Then evidently

$$
C_{\max }\left(\sigma_{\text {conc }}\right)=k a+2 \delta+(k+1) a .
$$

Thus we have

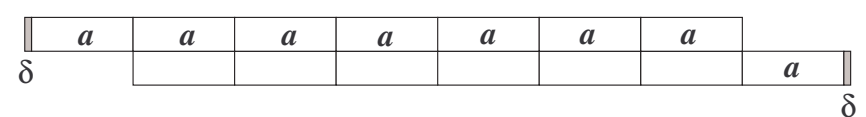

Figure 8: The optimal schedule.

$$
\frac{C_{\max }\left(\sigma_{\text {conc }}\right)}{C_{\max }^{*}}=\frac{k a+2 \delta+(k+1) a}{(k+1) a+2 \delta}=1+\frac{k a}{(k+1) a+2 \delta}
$$

which tends to 2 when $k$ tends to $\infty$. 


\section{References}

[1] A.A. Ageev and A.E. Baburin, Approximation algorithms for UET scheduling problems with exact delays. Oper. Res. Letters 35(2007), 533540 .

[2] A.A. Ageev and A.V. Kononov, Approximation algorithms for scheduling problems with exact delays. In: Approximation and Online Algorithms: 4th International Workshop (WAOA 2006), Zurich, Switzerland, LNCS 4368 (2007), 1-14.

[3] R.E. Burkard, V.G. Deineko, R. van Dal, J.A.A. van der Veen and G.J. Woeginger. Well-solvable special cases of the traveling salesman problem: A survey. SIAM Review, 40: 496-546, 1998.

[4] A. Condotta, Scheduling with due dates and time lags: new theoretical results and applications. Ph.D. Thesis, 2011, The University of Leeds, School of Computing, 156 pp.

[5] M. Elshafei, H. D. Sherali, and J.C. Smith, Radar pulse interleaving for multi-target tracking. Naval Res. Logist. 51 (2004), 79-94.

[6] P.C. Gilmore and R.E. Gomory. Sequencing a one-state variable machine: a solvable case of the traveling salesman problem. Operations Research 12 (1964), 655-679.

[7] P.C.Gilmore, E.L. Lawler and D.B. Shmoys. Well solved cases, in E.L. Lawler, J.K. Lenstra, A.H.G. Rinnooy Kan and D.B. Shmoys (Eds), The Traveling Salesman Problem: A Guided Tour of Combinatorial Optimization, Wiley, New York, pp. 87-143, 1986.

[8] J. Y.-T. Leung, H. Li, and H. Zhao, Scheduling two-machine flow shops with exact delays. International Journal of Foundations of Computer Science 18 (2007), 341-359.

[9] H. D. Sherali and J. C. Smith, Interleaving two-phased jobs on a single machine, Discrete Optimization 2 (2005), 348-361.

[10] W. Yu, The two-machine shop problem with delays and the one-machine total tardiness problem, Ph.D. thesis, Technische Universiteit Eindhoven, 1996.

[11] W. Yu, H. Hoogeveen, and J. K. Lenstra, Minimizing makespan in a two-machine flow shop with delays and unit-time operations is NP-hard. J. Sched. 7 (2004), no. 5, 333-348. 